\title{
BMJ Open Determinants of undergoing thyroid cancer screening in Korean women: a cross-sectional analysis from the K-Stori 2016
}

Ha Na Cho, ${ }^{1}$ Eunji Choi, ${ }^{1}$ Da Hea Seo, ${ }^{2}$ Boyoung Park, ${ }^{\circ}{ }^{3}$ Sohee Park, ${ }^{4}$ Juhee Cho, ${ }^{5}$ Sue Kim, ${ }^{6}$ Yeong-Ran Park, ${ }^{7}$ Yumie Rhee, ${ }^{8}$ Kui Son Choi ${ }^{1}$

To cite: Cho HN, Choi E, $\mathrm{Seo} \mathrm{DH}$, et al. Determinants of undergoing thyroid cancer screening in Korean women: a cross-sectional analysis from the K-Stori 2016. BMJ Open 2019;9:e026366. doi:10.1136/ bmjopen-2018-026366

- Prepublication history and additional material for this paper are available online. To view these files, please visit the journal online (http://dx.doi. org/10.1136/bmjopen-2018026366).

Received 29 August 2018 Revised 2 March 2019 Accepted 5 March 2019
Check for updates

(C) Author(s) (or their employer(s)) 2019. Re-use permitted under CC BY-NC. No commercial re-use. See rights and permissions. Published by BMJ.

For numbered affiliations see end of article.

Correspondence to

Dr Kui Son Choi;

kschoi@ncc.re.kr

\section{ABSTRACT}

Objectives Thyroid cancer is the most common cancer among Korean women. Studies suggest that the incidence of thyroid cancer might be associated with overdiagnosis resulting from thyroid cancer screening. The objective of this study was to identify the determinants of participation in thyroid cancer screening in Korean women.

Methods Data were obtained from the 2016 Korean Study of Women's Health-Related Issues, a nationwide crosssectional survey of women according to the reproductive life cycle. A total of 8697 cancer-free women of ages between 20 and 79 years were included for analysis. Multivariable logistic regression analysis was applied to analyse factors associated with adherence to thyroid cancer screening based on Andersen's health behavioural model.

Results Over the last 2 years, the rate of thyroid cancer screening was $39.2 \%$. In multivariable models, older age, higher household income, high school education level and higher perceived risk of cancer were positively associated with thyroid cancer screening participation. Moreover, women who underwent cervical cancer screening (adjusted OR [aOR] 3.67; 95\% Cl 2.90 to 4.64) and breast cancer screening (aOR $10.91 ; 95 \% \mathrm{Cl} 8.41$ to 14.14 ) had higher odds of attending thyroid cancer screening than women who did not attend cancer screening.

Conclusions These findings highlight the need to increase awareness of different recommendations on screening for various cancers to improve cost-effectiveness and to prevent unnecessary treatments.

\section{INTRODUCTION}

The incidence of thyroid cancer has increased globally over the last three decades. ${ }^{1}{ }^{2}$ Between 1999 and 2011, the age-standardised rate (ASR) of thyroid cancer incidence in Korea increased dramatically (annual percentage change $=22.8 \%){ }^{3}$ Although rates have decreased since then, about 25209 new thyroid cases $(11.7 \%$ of all malignancies) were registered in 2015: thyroid cancer (ASR, 55.6) was the most common cancer among Korean women, followed by breast (ASR, 49.2), colorectal (ASR, 22.2), stomach (ASR,

\section{Strengths and limitations of this study}

- This nationwide cross-sectional study documents the most recent rate of thyroid cancer screening among Korean women.

- Data were obtained from the 2016 Korean Study of Women's Health-Related Issues, a population-based, nationwide, cross-sectional survey for a reliable and representative research design.

- Experiences of thyroid cancer screening and factors associated with a cancer screening were assessed based on Andersen's behavioural model through face-to-face interviews.

- Due to cross-sectional study design, this study could not show the direction of causal relationships for the identified associations between thyroid cancer screening and other factors.

- More studies are needed to investigate trends in thyroid cancer screening rates and behaviours for both men and women in order to control current thyroid cancer epidemic issues in Korea.

20.5) and lung cancer (ASR, 14.3). ${ }^{3}$ Despite a steep increase in incidence, the age-standardised mortality rate of thyroid cancer remained stable, with a 5-year relative survival rate of $100.3 \% .^{3}$

Suggestive of an epidemic, increases in thyroid cancer incidence in Korea have been deemed the results of early detection of small papillary thyroid cancers, which progress slowly and pose a low risk of mortality. ${ }^{4}$ Indeed, previous studies reported that thyroid cancer screening rates in a region are strongly correlated with regional thyroid cancer incidence rates. ${ }^{5}$ The apparent thyroid cancer epidemic was also explained to be the result of increases in the incidence of small tumours. ${ }^{7}$ All of these studies strongly raised the possibility of overdiagnosis caused by thyroid cancer screening.

In Korea, the National Cancer Screening Program (NCSP), which was implemented 
in 1999, provides nationwide screening services free of charge or with a small copayment for gastric, liver, colorectal, breast and cervical cancers. ${ }^{8}$ Although thyroid cancer screening is not covered under the NCSP, healthcare providers typically offer ultrasonographic screening for thyroid cancer as an inexpensive add-on at around US\$30-US\$50. ${ }^{9}$ In 2015, a multidisciplinary expert committee was organised to establish recommendations for thyroid cancer screening. This committee determined that thyroid ultrasonography screening should not be routinely recommended for healthy subjects, as current evidence with which to assess the balance of benefits and harms of thyroid cancer screening by ultrasonography is insufficient. ${ }^{10}$ Through these efforts, there has been a marked decrease in thyroid operations. Also, the age-standardised incidence rates for thyroid cancer have decreased swiftly. ${ }^{3} 11$

Notwithstanding, the incidence of thyroid cancer in Korea still remains extraordinarily high, especially in women. Thyroid cancer is the most commonly diagnosed cancer in women, and incidence rates are more than 3.6 times higher than those of men. ${ }^{3}$ Thus, public concern was raised concerning the potential causes of thyroid cancer, especially in women. However, few studies on thyroid cancer screening behaviours in Korea women have been undertaken. ${ }^{12}$ Thus, this study was designed to identify factors associated with thyroid cancer screening participation in Korean women based on Anderson's behavioural model.

Anderson's behavioural model has served as a guide in the selection of relevant variables with which to analyse determinants of the healthcare people receive since $1968 .{ }^{14}$ The model is widely used in various settings for primary healthcare, general healthcare, outpatient utilisation and services in specific settings, such as mental and oral healthcare. ${ }^{15}$ This framework has also been used to identify factors associated with cancer screening utilisation. ${ }^{16-19}$ The model is a multilevel model that includes both individual factors and contextual determinants of healthcare use. ${ }^{20}$ According to this model, an individual's use of health services is determined by three main components, including predisposing factors, enabling factors and need factors. ${ }^{16}$ Based on previous research, Anderson's behavioural model was chosen as the best framework for identifying the determinants of thyroid cancer screening attendance. Accordingly, we chose this model to assess thyroid cancer screening rates and to identify determinants of thyroid cancer screening adherence in Korean women.

\section{MATERIALS AND METHODS \\ Study population}

This study was based on the Korean Study of Women's Health-Related Issues (K-Stori) 2016. The K-Stori is a nationwide survey designed to investigate broad health issues among Korean women according to five stages in the life cycle of women (adolescence, 14-17 years; childbearing, 19-44 years; pregnancy and Post partum, 19-44 years; menopause, 45-64 years and older adulthood, 65-79 years). ${ }^{21}$ We randomly sampled 3000 women in each stage of the life cycle for a reliable and representative research design. For random sampling of a multilevel, stratified, probability proportion, a statistics extraction method was used as a sampling framework using the 2010 Population and Housing Census.

Trained interviewers from a professional research agency conducted door-to-door surveys to assess study eligibility. A total of 15000 women aged 14-79 years completed in-person interview surveys (adolescents completed online surveys) between April 2016 and June 2016. The response rate of K-Stori was $40.4 \%$. The detailed methods of this survey have been explained elsewhere. ${ }^{21}$ Of the 15000 women who participated in the K-Stori survey, we asked about thyroid cancer screening only for women in childbearing, menopause and older adulthood stages. We did not ask women in the adolescence and pregnancy and postpartum stages about thyroid cancer screening, because they are not appropriate for assessing cancer screening behaviour: women in adolescence are too young to assess cancer screening behaviour, and women in pregnancy and post partum are pregnant or have had a birth within less than 1 year. Thus, 6000 women in the adolescence and pregnancy and postpartum stages of life cycle were excluded. Also, 94 women aged 19 years and 209 women who answered that they had been diagnosed with cancer or thyroid disease were excluded. The final study subjects comprised 8697 women aged 20-79 years.

\section{Questionnaires}

Study participants were asked about their experiences with health examinations, including cancer screening. Women who answered 'yes' to receiving a health examination were then asked whether they had undergone thyroid cancer screening during the last 2 years.

Factors associated with a cancer screening were assessed via Andersen's behavioural model. ${ }^{14}{ }^{15}$ Final variables were selected from literature reviews of previous studies related to determinants of cancer screening. ${ }^{16-19}$ Age (20-29, 30-39, 40-49, 50-59, 60-69 and 70-79), marital status (unmarried, married, divorced/separated), family size (live alone, two to three, four or more people), education level (elementary school or less, middle/high school, university and higher), employment status (unemployed, employed) and health screening belief (weak, moderate, strong) were included as predisposing factors. As enabling factors, we included household income ( $\leq$ US $\$ 1699$, US $\$ 1700-$ US $\$ 3499$, $\geq$ US $\$ 3500$ ), private insurance (yes, no), residence area (urban, rural) and accessibility to medical services (yes, no). Finally, self-reported health status (good, average, bad), perceived risk of cancer (low, average, high), chronic disease (yes, no) and other cancer screening behaviours (cervical cancer screening in past 2 years; breast cancer screening in past 2 years) were considered as need factors. 


\section{Statistical analyses}

$\mathrm{X}^{2}$ analysis was used to determine the statistical significance of thyroid cancer screening behaviours according to variables selected as predisposing factors, enabling factors and need factors. Since 3000 individuals were sampled for each life cycle, calculating weighted cancer screening rate was needed based on age groups as 8697 women aged 20-79 years were included in the study. Survey weighting adjustments were applied to calculate cancer screening rates in a representative population of Korean women. In addition, in multivariable logistic regression analysis, we applied age-adjusted weighting values for women in Korea. Multivariable logistic regression analysis was conducted in sequential blocks to analyse associations among predisposing factors, enabling resources and health needs in relation to thyroid cancer screening. Model 1 included predisposing factors only; model 2 added enabling factors to model 1 and model 3 added need factors. All reported ORs were considered significant if $p<0.05$. Data were analysed using SAS V.9.3 (SAS Institute).

\section{Patient involvement}

No patients were involved in this study.

\section{RESULTS}

Among 8697 women aged $20-79$ years, $41.6 \%$ had undergone thyroid cancer screening within the last 2 years (table 1). In terms of predisposing factors, women aged $50-59$ years $(58.0 \%)$, married women $(48.0 \%)$, women with a family size of two to three members $(46.3 \%)$, women with a middle/high school education (48.2\%) and women who had weak belief in the effectiveness of health screening $(43.8 \%)$ were significantly more likely to undergo thyroid cancer screening. Regarding enabling factors, women who lived in the rural area (43.9\%), had private insurance $(43.9 \%)$ and had no experiences of unmet healthcare needs $(42.0 \%)$ were more likely to undergo thyroid cancer screening. In terms of need factors, more women who reported that their health status was average $(45.4 \%)$, had a higher perceived risk of cancer $(46.2 \%)$, responded as previously or currently having chronic diseases $(48.7 \%)$ and had previously undergone cervical cancer screening $(64.4 \%)$ or breast cancer screening $(65.5 \%)$ attended thyroid cancer screening.

Within the last 2 years, the weighted thyroid cancer screening rate was $39.2 \%$. Women aged $50-59$ years exhibited the highest rate of thyroid cancer screening (57.0\%), whereas women aged 20-29 years held the lowest thyroid cancer screening rate $(11.0 \%)$. Along with thyroid cancer screening, breast and cervical cancer screening rates showed similar trends according to age groups (figure 1 ). Figure 2 depicts the results of our analysis of correlations between thyroid cancer screening rates and other cancer screening rates according to age. Therein, we noted a strong positive correlation between thyroid cancer screening rate and screening for other cancers (breast: $\mathrm{r}=0.9558, \mathrm{p}<0.0001$, cervical: $\mathrm{r}=0.9232, \mathrm{p}<0.0001$ ) with significant results.

Table 2 lists the factors associated with thyroid cancer screening according to Andersen's behavioural model. We conducted multivariable logistic regression analysis with predisposing factors only in model 1 , with the addition of enabling factors in model 2 , and with the addition of need factors in model 3. In model 1, compared with women of ages 40-49 years, those aged less than 40 years were less likely to undergo thyroid cancer screening (20-29: adjusted OR [aOR] 0.26; 95\% CI 0.19 to $0.35,30-39$ : aOR $0.58 ; 95 \%$ CI 0.47 to 0.71 ), whereas women older than 50 years were more likely to undergo thyroid cancer screening (aOR 1.78; 95\% CI 1.48 to 2.13). Married women had the highest odds of undergoing thyroid cancer screening, compared with women who were not married (aOR 1.74; 95\% CI 1.33 to 2.29). Women with higher education showed increased odds of attending thyroid cancer screening (middle/high school: aOR 1.22; $95 \%$ CI 1.05 to 1.43 , university and higher: aOR $1.26 ; 95 \%$ CI 1.02 to 1.55 ). Interestingly, women who had a strong belief in the effectiveness of health screening had lower odds of attending thyroid cancer screening than women with weak belief (aOR 0.79; $95 \%$ CI 0.68 to 0.91 ). Employed women were more likely to undergo thyroid cancer screening than those who were unemployed (aOR 1.19; 95\% CI 1.05 to 1.34).

In model 2, in which we added enabling factors to model 1, age group, marital status and belief in the effectiveness of health screening showed similar results to those attained in model 1. Meanwhile, larger family size additionally showed a negative association with attending thyroid cancer screening. Regarding enabling factors, women with a household income equal to or more than US $\$ 3500$ had higher odds of attending thyroid cancer screening than women with income equal to or less than US $\$ 1699$ (aOR $1.55 ; 95 \%$ CI 1.28 to 1.89 ). Women who had private insurance had higher odds of attending thyroid cancer screening than women who did not have private insurance (aOR $1.45 ; 95 \%$ CI 1.24 to 1.69 ). An experience of an unmet healthcare need did not significantly alter results (aOR $0.99 ; 95 \%$ CI 0.81 to 1.21 ).

In model 3 , after adding need factors to model 2, women of ages of 50-59 (aOR 1.58; 95\% CI 1.28 to 1.94 ), 60-69 (aOR 1.64; 95\% CI 1.27 to 2.10) and 70-79 (aOR 2.27; $95 \%$ CI 1.68 to 3.07 ) years were significantly more likely to undergo thyroid cancer screening, compared with women aged $40-49$ years. Women who had a middle or high school education, moderate belief in the effectiveness of health screening, household income equal to or more than US $\$ 3500$, and higher perceived risk of cancer were more likely to attend thyroid cancer. Attending breast or cervical cancer screening was the strongest predictor for undergoing thyroid cancer screening. Women who attended cervical cancer screening (aOR 3.67; 95\% CI 2.90 to 4.64 ) and breast cancer screening (aOR 10.91; $95 \%$ CI 8.41 to 14.14) had higher odds of attending thyroid 
Table 1 Characteristics of the study subjects according to receipt of thyroid cancer screening within 2 years $(n=8697)$

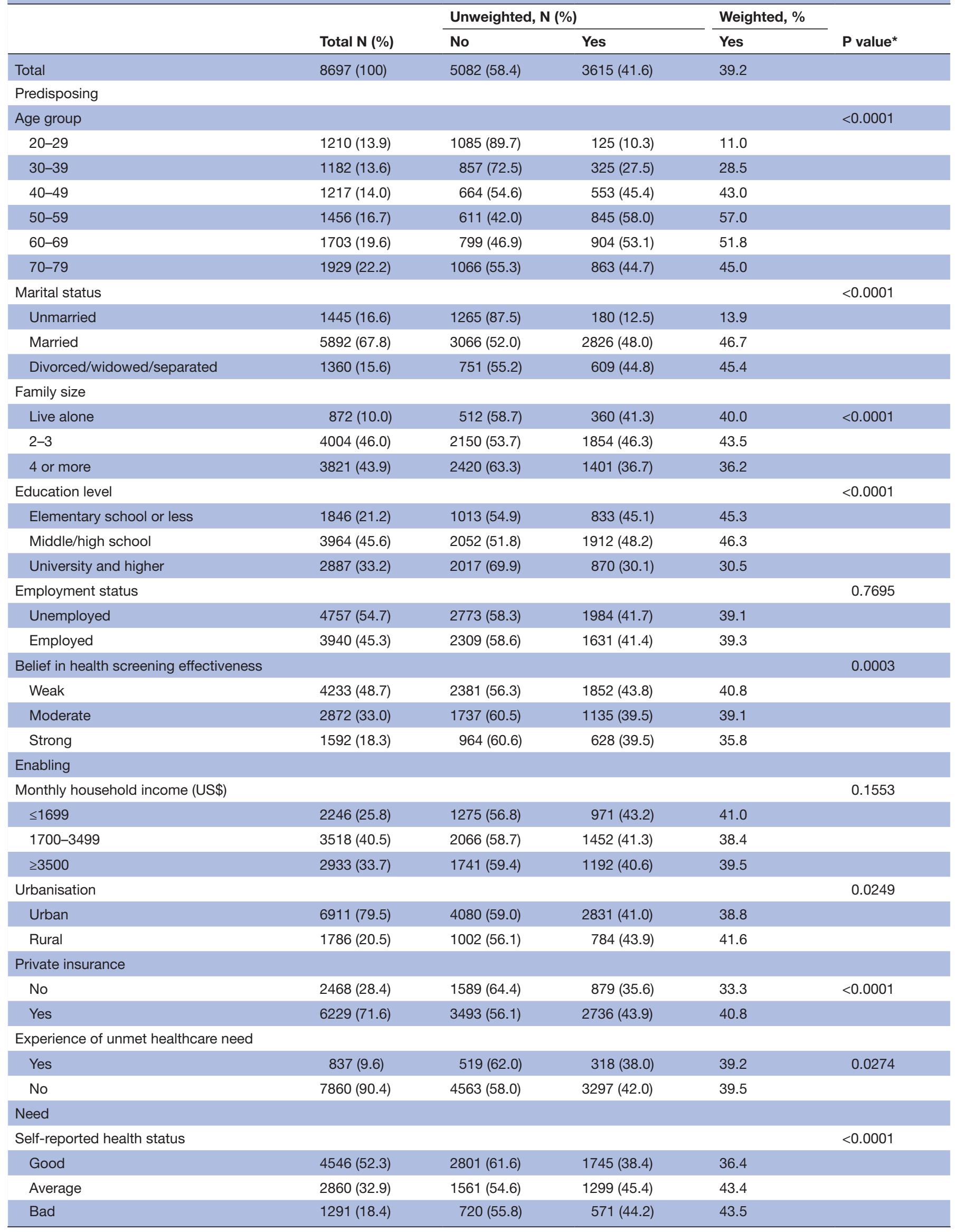




\section{Table 1 Continued}

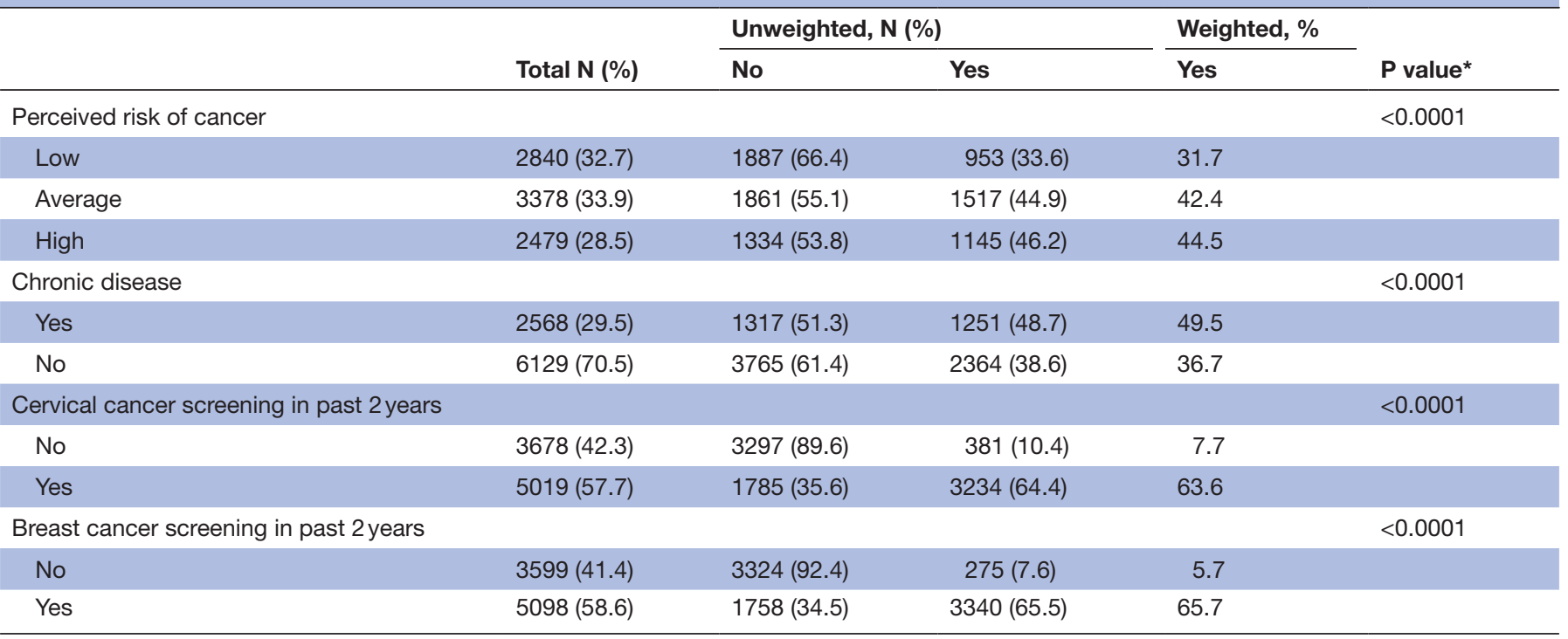

${ }^{*} \mathrm{P}$ value for unweighted thyroid cancer screening rates.

cancer screening than women who had not undergone screening for either of these other cancers.

\section{DISCUSSION}

This study examined recent thyroid cancer screening rates and factors associated with thyroid cancer screening over the last 2 years for women aged 20-79 years according to Andersen's behavioural model. Even though Korean cancer guidelines do not recommend thyroid cancer screening for asymptomatic individuals, the weighted rate of thyroid cancer screening among women was $39.2 \%$, which the highest it has been in Korea. Thyroid cancer screening rates for women were reported at $16.4 \%$ by the National Cancer Screening Survey in 2009, at 16\% by the Korean Community Health Survey in 2010 and at $31.3 \%$ by the National Evidence-Based Healthcare Collaborating Agency in 2011. ${ }^{6} 1213$ These three studies were all nationwide surveys, although they used different questionnaires, age ranges and screening intervals. Due to these differences, we could not confirm an increase in thyroid cancer screening rates by comparing our results with previous studies. However, it can be inferred that the rate of screening has increased.

Studies in Korea are reporting correlations between increased incidences of and screening rates for thyroid cancer. ${ }^{79}$ In the present study, we noted that more than

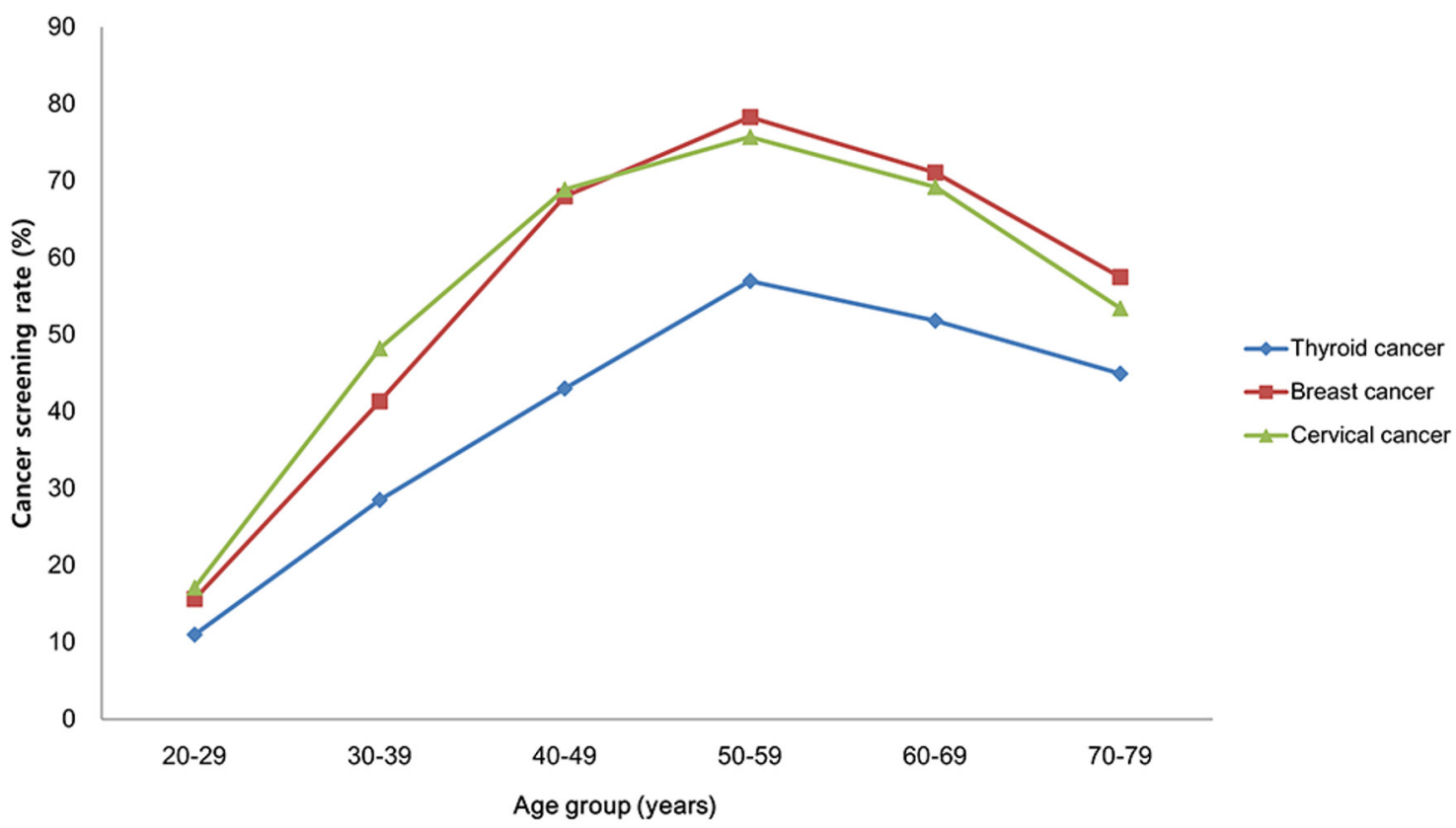

Figure 1 Weighted cancer screening rates for three types of cancer according to age groups. 

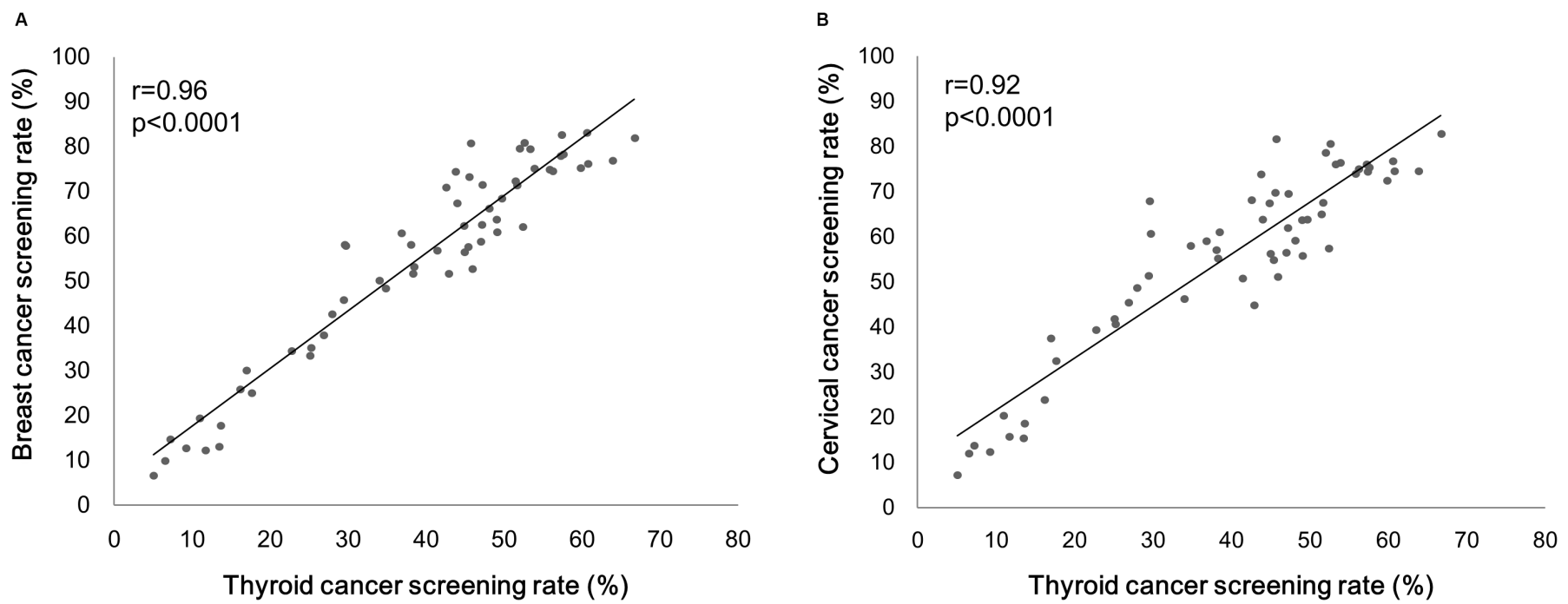

Figure 2 Correlations between thyroid cancer screening rate and $(A)$ breast and $(B)$ cervical cancer screening rate according to age (a year). Each dot represents age.

half of women aged 50-59 (57.0\%) and 60-69 (51.8\%) years attended thyroid cancer screening over the last 2 years. Consistent with these results, previous studies have also indicated that women aged $50-59$ years exhibit the highest thyroid cancer screening rate. ${ }^{12} 22$ Meanwhile, the age-specific incidence of thyroid cancer in 2013 peaked in Korean women aged 50-59 years. ${ }^{7}$ Additionally, when we compared thyroid cancer incidence in data from the Korean Central Cancer Registry in 2014 and thyroid cancer screening rates from our study, we noted strong correlations between them (online supplementary 1). These results support the association between thyroid cancer incidence and thyroid cancer screening. Indeed, many hospitals and clinicians in Korea encourage routine health checks that include the option of screening for thyroid cancer (for an additional fee, as this is not covered by national health insurance).$^{67}$

As factors associated with thyroid cancer screening in multivariable logistic regression analysis, women with older age, middle or high school education level, moderate belief in health screening effectiveness, household income equal to or more than US $\$ 3500$, average or high levels of perceived risk of cancer and prior experience with breast or cervical cancer screening were more likely to undergo thyroid cancer screening. Women who attended cervical cancer screening (aOR 3.67; 95\% CI 2.90 to 4.64 ) or breast cancer screening (aOR 10.91; 95\% CI 8.41 to 14.14 ) had higher odds of attending thyroid cancer screening than women who did not attend cancer screening. Our results are similar to those in previous studies on the association between participation in screening for other cancers and thyroid cancer screening. ${ }^{12}{ }^{13}$ In particular, it has been reported that women who have undergone breast cancer screening are prone to undergo thyroid cancer screening. ${ }^{1718}$

With the promotion of cancer screening by the government and media, participation rates in the NCSP have increased dramatically over the last two decades. ${ }^{8} 23$
Although thyroid cancer screening is not included in the NCSP, the rate of screening for thyroid cancer seems to have been affected by the NCSP. In general, people who participate the NCSP can receive thyroid cancer screening additionally based on their own needs or their physician's recommendations by paying an extra fee. In support of this notion, researchers reported that, in Korea, the incidence of thyroid cancer increased slowly beginning in 1990; however, after the NCSP was launched for gastric, breast, cervical, liver and colorectal cancer, it rapidly increased. ${ }^{8}$ According to cancer statistics, the incidence of thyroid cancer in 2011 was 15-fold higher than that in $1993 .^{9}$

In recent decades, the incidence of thyroid cancer has increased steadily and consistently in many developed countries. According to a recently reported article, the highest rates ( $>25$ cases per 100000 women) were observed in Korea, Israel, Canada, USA, Italy, France and low-income and middle-income countries (LMICs), such as Turkey, Costa Rica, Brazil and Ecuador. ${ }^{24}$ This international comparison study confirmed the very high thyroid cancer incidence rates in some, but not all, high-income countries in 2008-2012 and also showed very high incidence rates in several LMICs, particularly in urban areas. ${ }^{24}$ The very high thyroid cancer incidence and low mortality rates in some LMICs also strongly suggest a major role for overdiagnosis. Ultrasound examinations have become increasingly available, and encouraging opportunistic screening has increased detection of smaller, asymptomatic nodules. Overdiagnosis is more likely to occur under privately oriented healthcare systems in the absence of a gatekeeping role in primary care for referral to secondary care and under systems where doctors are paid by a fee for service. Also, the absence of thyroid cancer screening guidelines may contribute diagnosis of indolent tumours. Thus, special concern is needed for these countries to reduce overdiagnosis. 
Table 2 Associations among predisposing, enabling and need factors in relation to thyroid cancer screening within the last 2 years $(n=8697)$

\begin{tabular}{|c|c|c|}
\hline Model 1 & Model 2 & Model 3 \\
\hline $95 \% \mathrm{Cl}$ & $95 \% \mathrm{Cl}$ & $95 \% \mathrm{Cl}$ \\
\hline
\end{tabular}

Predisposing

Age group

\begin{tabular}{|c|c|c|c|c|c|c|}
\hline 20-29 & 0.26 & (0.19 to 0.35$)$ & 0.26 & (0.19 to 0.37$)$ & 0.71 & (0.51 to 1.01$)$ \\
\hline $30-39$ & 0.58 & (0.47 to 0.71$)$ & 0.60 & (0.48 to 0.73 ) & 1.01 & (0.79 to 1.28$)$ \\
\hline $40-49$ & 1.00 & & 1.00 & & 1.00 & \\
\hline $60-69$ & 1.53 & (1.25 to 1.88$)$ & 1.62 & (1.32 to 1.98$)$ & 1.64 & (1.27 to 2.10$)$ \\
\hline $70-79$ & 1.30 & (1.04 to 1.62$)$ & 1.51 & (1.19 to 1.90$)$ & 2.27 & (1.68 to 3.07$)$ \\
\hline Divorced/widowed/separated & 1.52 & (1.10 to 2.11$)$ & 1.58 & (1.14 to 2.21$)$ & 1.07 & (0.74 to 1.55$)$ \\
\hline Married & 1.74 & (1.33 to 2.29$)$ & 1.73 & (1.31 to 2.28$)$ & 0.88 & (0.65 to 1.20$)$ \\
\hline \multicolumn{7}{|l|}{ Family size } \\
\hline Live alone & 1.00 & & 1.00 & & 1.00 & \\
\hline Elementary school or less & 1.00 & & 1.00 & & 1.00 & \\
\hline Middle/high school & 1.22 & (1.05 to 1.43$)$ & 1.07 & (0.91 to 1.26$)$ & 1.28 & (1.02 to 1.59$)$ \\
\hline University and higher & 1.26 & (1.02 to 1.55$)$ & 0.99 & (0.80 to 1.24$)$ & 1.27 & (0.96 to 1.68$)$ \\
\hline \multicolumn{7}{|l|}{ Employment status } \\
\hline Unemployed & 1.00 & & 1.00 & & 1.00 & \\
\hline Employed & 1.19 & (1.05 to 1.34$)$ & 1.1 & (0.97 to 1.24$)$ & 0.91 & (0.79 to 1.06$)$ \\
\hline \multicolumn{7}{|c|}{ Belief of health screening effectiveness } \\
\hline Weak & 1.00 & & 1.00 & & 1.00 & \\
\hline Moderate & 0.99 & (0.87 to 1.12$)$ & 1.00 & (0.87 to 1.13$)$ & 1.25 & (1.07 to 1.45$)$ \\
\hline
\end{tabular}

Monthly household income (US\$)

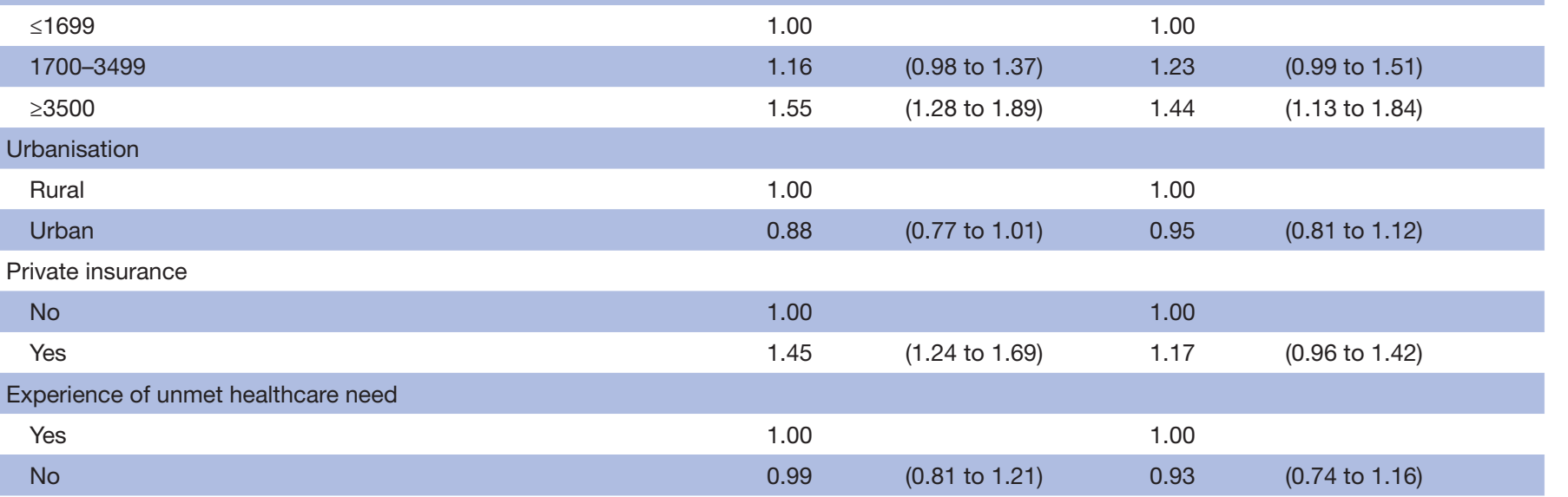

Need

Self-reported health status

$\begin{array}{lrr}\text { Good } & 1.00 & \\ \text { Average } & 0.84 & (0.65 \text { to } 1.09) \\ \text { Bad } & 0.96 & (0.82 \text { to } 1.12)\end{array}$

Continued 
Table 2 Continued

\begin{tabular}{|c|c|c|c|c|c|c|}
\hline & \multicolumn{2}{|c|}{ Model 1} & \multicolumn{2}{|c|}{ Model 2} & \multicolumn{2}{|c|}{ Model 3} \\
\hline & aOR & $95 \% \mathrm{Cl}$ & aOR & $95 \% \mathrm{Cl}$ & aOR & $95 \% \mathrm{Cl}$ \\
\hline \multicolumn{7}{|c|}{ Perceived risk of cancer } \\
\hline Average & & & & & 1.21 & (1.03 to 1.42$)$ \\
\hline High & & & & & 1.27 & (1.06 to 1.53$)$ \\
\hline \multicolumn{7}{|c|}{ Chronic disease } \\
\hline Yes & & & & & 1.13 & (0.93 to 1.38$)$ \\
\hline \multicolumn{7}{|c|}{ Cervical cancer screening in past 2 years } \\
\hline No & & & & & 1.00 & \\
\hline Yes & & & & & 3.67 & (2.90 to 4.64$)$ \\
\hline
\end{tabular}

Model 1: Adjusted with predisposing factors. Model 2: Adjusted with predisposing and enabling factors. Model 3: Adjusted with predisposing, enabling and need factors.

aOR, adjusted OR.

In previous studies, socioeconomic status was identified as a significant risk factor affecting the prevalence of thyroid cancer. ${ }^{225}$ In one of these studies, women with higher levels of household income were found to have attended thyroid cancer screening more. ${ }^{25}$ This is consistent with our study. In Korea, thyroid cancer screening is provided via opportunistic screening, ${ }^{13}$ and opportunistic screening is more commonly used by people with higher income in Korea. ${ }^{26}$ Meanwhile, we also found perceived risk of cancer to be a significant factor in thyroid cancer screening attendance. While there are no studies related to perceived cancer risk and thyroid cancer screening, a significant association between breast cancer risk and mammography has been reported. ${ }^{27}$ We suspect that recent reports of steep increases in thyroid cancer incidence may have increased fears and perceived risk of thyroid cancer.

The current study reports the most recent thyroid cancer screening rate and factors associated therewith in women using data from a nationwide survey. This was the first study to focus on women's health-related issues concerning thyroid cancer screening. However, there are some limitations to this study. Due to the cross-sectional study design, this study could not show the direction of causal relationships for the identified associations between thyroid cancer screening and other factors. Also, information on thyroid cancer screening and other independent variables could contribute to recall bias because the data were obtained from a self-reported survey (K-Stori). Previous research has shown that screening rates from self-reported surveys can be under or overestimated depending on individual characteristics. ${ }^{28}{ }^{29}$ Notwithstanding, the survey was conducted by trained interviewers, and professional research agencies provided quality control for interviewers and ensured missing values and data errors were minimal.
Although this study reports current thyroid cancer screening rates and factors associated therewith, further studies are needed to investigate and to control current thyroid cancer epidemic issues in Korea. In particular, since the National Cancer Screening Guidelines only recently announced that thyroid cancer screening is not recommended in asymptomatic adults, it is necessary to assess changes in thyroid cancer screening behaviour as a result thereof. Also, further study is needed to determine potential causes of thyroid cancer and thyroid cancer screening behaviour for both men and women.

\section{CONCLUSIONS}

In conclusion, even though thyroid cancer screening is not recommended for asymptomatic populations, about $40 \%$ of Korean women attended thyroid cancer screening over the last 2years. Women who attended breast or cervical cancer, which are nationally recommended for Korean women, were more likely to attend thyroid cancer screening. These findings highlight the need to increase awareness of different recommendations for various cancer screenings in order to minimise poor cost-effectiveness and risk of overdiagnosis. Increasing awareness of the benefits and risks of thyroid cancer screening may require targeted intervention.

\section{Author affiliations}

${ }^{1}$ Graduate School of Cancer Science and Policy, National Cancer Center, Goyang, Korea (the Republic of)

${ }^{2}$ Department of Endocrinology and Metabolism, Inha University College of Medicine, Incheon, Korea (the Republic of)

${ }^{3}$ College of Medicine, Hanyang University, Seoul, Korea (the Republic of)

${ }^{4}$ Graduate School of Public Health, Yonsei University, Seoul, Korea (the Republic of) 
${ }^{5}$ Department of Clinical Research and Evaluation, Sungkyunkwan University, Seoul, Korea (the Republic of)

${ }^{6}$ College of Nursing, Yonsei University, Seoul, Korea (the Republic of)

${ }^{7}$ Division of Silver Industry, Kangnam University, Yongin, Korea (the Republic of)

${ }^{8}$ Division of Endocrinology, Department of Internal Medicine, Yonsei University

College of Medicine, Seoul, Korea (the Republic of)

Contributors All authors were involved conceptualisation and design of the study. HNC contributed to drafting and writing the manuscript. HNC, EC, DHS, SP, JC and $\mathrm{BP}$ contributed to developing the questionnaire, revising the article and conducting data analysis. SK and Y-RP contributed to editing, reviewing and final approval of the article. KSC and YR contributed to the revision of the manuscript and design and supervision of the study. All authors read and approved the final manuscript.

Funding This research was supported by a fund from the Research of Korea Centers for Disease Control and Prevention (\#2015ER630300) and a Grantin-Aid for Cancer Research and Control from the National Cancer Center of Korea (\#1610401).

Competing interests None declared.

Patient consent for publication Not required.

Ethics approval This study was approved by the Institutional Review Board of the National Cancer Center, Korea (NCC2016-0062).

Provenance and peer review Not commissioned; externally peer reviewed.

Data sharing statement The data collected from the study are owned by research funding agencies (KCDC). Thus, the official request channel for datasets used and/or analysed in the current study is the Korea Centers for Disease Control and Prevention. Thus, the datasets used and/or analysed during the current study are available from the Korea Centers for Disease Control and Prevention upon reasonable request. The contact information for access is as follows; Division of cardiovascular Diseases, Center for Biomedical Science Korea National Institute of Health, Korea Center for Disease Control \& Prevention wnddus7361@korea.kr.

Open access This is an open access article distributed in accordance with the Creative Commons Attribution Non Commercial (CC BY-NC 4.0) license, which permits others to distribute, remix, adapt, build upon this work non-commercially, and license their derivative works on different terms, provided the original work is properly cited, appropriate credit is given, any changes made indicated, and the use is non-commercial. See: http://creativecommons.org/licenses/by-nc/4.0/.

\section{REFERENCES}

1. Ferlay J, Bray F, Steliarova-Foucher E, et al. Cancer incidence in five continents, CI5plus. IARC CancerBase 2014.

2. Pellegriti G, Frasca F, Regalbuto C, et al. Worldwide increasing incidence of thyroid cancer: update on epidemiology and risk factors. J Cancer Epidemiol 2013;2013:1-10.

3. Jung KW, Won YJ, Kong HJ, et al. Cancer statistics in korea: incidence, mortality, survival, and prevalence in 2015. Cancer Res Treat 2018;50:303-16.

4. Cooper DS, Doherty GM, Haugen BR, et al. Revised American Thyroid Association management guidelines for patients with thyroid nodules and differentiated thyroid cancer. Thyroid 2009;19:1167-214.

5. Ahn HY, Park YJ. Incidence and clinical characteristics of thyroid cancer in Korea. Korean Journal of Medicine 2009;77:537-42.

6. Ahn HS, Kim HJ, Kim KH, et al. Thyroid cancer screening in South Korea increases detection of papillary cancers with no impact on other subtypes or thyroid cancer mortality. Thyroid 2016;26:1535-40.

7. Park S, Oh CM, Cho H, et al. Association between screening and the thyroid cancer "epidemic" in South Korea: evidence from a nationwide study. BMJ 2016;355:i5745.
8. Kim Y, Jun JK, Choi KS, et al. Overview of the National Cancer screening programme and the cancer screening status in Korea. Asian Pac J Cancer Prev 2011;12:725-30.

9. Ahn HS, Kim HJ, Welch HG. Korea's thyroid-cancer "epidemic"-screening and overdiagnosis. N Engl J Med 2014;371:1765-7.

10. Yi KH, Kim SY, Kim DH, et al. The Korean guideline for thyroid cancer screening. Journal of the Korean Medical Association 2015;58:302-12.

11. Ahn HS W. South Korea's thyroid-cancer "epidemic"-turning the tide. 2015;373:2389-90.

12. Han MA, Choi KS, Lee HY, et al. Current status of thyroid cancer screening in Korea: results from a nationwide interview survey. Asian Pac J Cancer Prev 2011;12:1657-63.

13. Kim SY, Jang BH, Eea S. A research on evidence based healthcare for thyroid cancer screening. Seoul Korea: National Evidence Based Healthcare Collaborating Agency (NECA), 2012.

14. Andersen R, Newman JF. Societal and individual determinants of medical care utilization in the United States. Milbank Mem Fund Q Health Soc 1973;51:95-124.

15. Babitsch B, Gohl D, von Lengerke T. Re-revisiting andersen's behavioral model of health services use: a systematic review of studies from 1998-2011. GMS Psycho-Social-Medicine 2012:9.

16. Park $\mathrm{H}$. Factors associated with utilization of uterine cervical cancer screening services by Korean Women using the Andersen Behavioral Model. Life Science Journal 2014;11.

17. Vyas A, Madhavan S, LeMasters T, et al. Factors influencing adherence to mammography screening guidelines in Appalachian women participating in a mobile mammography program. $J$ Community Health 2012;37:632-46.

18. Couture MC, Nguyen CT, Alvarado BE, et al. Inequalities in breast and cervical cancer screening among urban Mexican women. Prev Med 2008:47:471-6.

19. Park YS, Chung EK, Choi JS, et al. Cancer screening rates and its related factors in a rural and urban communities. Journal of the Korean Academy of Family Medicine 2006;27:21-32.

20. Andersen RM. Revisiting the behavioral model and access to medical care: does it matter? J Health Soc Behav 1995:36:1.

21. Cho HN, Choi E, Seo DH, et al. The Korean Study of Women's Health-Related Issues (K-Stori): rationale and study design. BMC Public Health 2017;17:609.

22. Kim JM, Kim HM, Jung BY, et al. The association between cancer incidence and family income: analysis of Korean National Health Insurance cancer registration data. Asian Pac J Cancer Prev 2012;13:1371-6.

23. Suh M, Choi KS, Park B, et al. Trends in cancer screening rates among Korean men and women: results of the Korean National Cancer Screening Survey, 2004-2013. Cancer Research and Treatment 2016;48:1-10.

24. Lortet-Tieulent J, Franceschi S, Dal Maso L, et al. Thyroid cancer "epidemic" also occurs in low-and middle-income countries. 2018.

25. Choi SW, Ryu SY, Han MA, et al. The association between the socioeconomic status and thyroid cancer prevalence; based on the Korean National Health and Nutrition Examination Survey 2010-2011. $J$ Korean Med Sci 2013;28:1734-40.

26. Kang S, You CH, Kwon YD. [The determinants of the use of opportunistic screening programs in Korea]. J Prev Med Public Health 2009;42:177-82.

27. Katapodi MC, Lee KA, Facione NC, et al. Predictors of perceived breast cancer risk and the relation between perceived risk and breast cancer screening: a meta-analytic review. Prev Med 2004;38:388-402.

28. McPhee SJ, Nguyen TT, Shema SJ, et al. Validation of recall of breast and cervical cancer screening by women in an ethnically diverse population. Prev Med 2002;35:463-73.

29. Vacek PM, Mickey RM, Worden JK. Reliability of self-reported breast screening information in a survey of lower income women. Prev Med 1997;26:287-91. 